\title{
The association of lesion eccentricity with plaque morphology and components in the superficial femoral artery: a high-spatial-resolution, multi-contrast weighted CMR study
}

\author{
Feiyu Li1,2, Mary McGrae McDermott 3 , Debiao Li4, Timothy J Carroll4, Daniel S Hippe2, Christopher M Kramer ${ }^{5}$, \\ Zhaoyang Fan4, Xihai Zhao², Thomas S Hatsukami6, Baocheng Chu², Jinnan Wang7 and Chun Yuan*2
}

\begin{abstract}
Background: Atherosclerotic plaque morphology and components are predictors of subsequent cardiovascular events. However, associations of plaque eccentricity with plaque morphology and plaque composition are unclear. This study investigated associations of plaque eccentricity with plaque components and morphology in the proximal superficial femoral artery using cardiovascular magnetic resonance (CMR).

Methods: Twenty-eight subjects with an ankle-brachial index less than 1.00 were examined with 1.5T high-spatialresolution, multi-contrast weighted CMR. One hundred and eighty diseased locations of the proximal superficial femoral artery (about $40 \mathrm{~mm}$ ) were analyzed. The eccentric lesion was defined as [(Maximum wall thickness- Minimum wall thickness)/Maximum wall thickness] $\geq 0.5$. The arterial morphology and plaque components were measured using semi-automatic image analysis software.

Results: One hundred and fifteen locations were identified as eccentric lesions and sixty-five as concentric lesions. The eccentric lesions had larger wall but similar lumen areas, larger mean and maximum wall thicknesses, and more calcification and lipid rich necrotic core, compared to concentric lesions. For lesions with the same lumen area, the degree of eccentricity was associated with an increased wall area. Eccentricity (dichotomous as eccentric or concentric) was independently correlated with the prevalence of calcification (odds ratio $3.78,95 \% \mathrm{Cl} 1.47-9.70$ ) after adjustment for atherosclerotic risk factors and wall area.
\end{abstract}

Conclusions: Plaque eccentricity is associated with preserved lumen size and advanced plaque features such as larger plaque burden, more lipid content, and increased calcification in the superficial femoral artery.

\section{Background}

Peripheral arterial disease (PAD), a chronic atherosclerotic occlusive disease in the lower extremities, affects 8 to 12 million Americans [1,2]. Local symptoms include intermittent claudication, skin ulcer and progressive extremity gangrene [3]. The risk of death for PAD patients with claudication, especially from coronary and cerebrovascular events, is approximately 2 times greater than the risk for controls without PAD [2,4]. Despite their increased risk of cardiovascular events, patients with

\footnotetext{
* Correspondence: cyuan@u.washington.edu

2 Department of Radiology, University of Washington, Seattle, WA, USA Full list of author information is available at the end of the article
}

PAD are frequently underdiagnosed and undertreated [1].

Previous studies in various arterial beds have demonstrated that plaque compositions are strongly associated with plaque progression, vulnerability and subsequent symptoms [5-7]. Plaques that show intraplaque hemorrhage (IPH), thinned or ruptured fibrous caps and larger lipid rich necrotic cores (LRNC), are more likely to cause cardiovascular events $[5,6]$. The lower extremity arterial calcification content, measured by computed tomography, has been demonstrated as a potential marker for PAD severity and high risk of amputation in PAD patients [7]. 
Besides plaque composition, a number of identified morphological characteristics also provide important prognostic information for atherosclerotic lesions, one of which is lesion eccentricity [8]. A few studies suggested that the patients with cerebrovascular and cardiovascular symptoms trended to present with eccentric plaques rather than concentric lesions $[9,10]$. In addition, the progression of atherosclerosis disease was identified more common in eccentric lesions as compared to concentric geometry [11].

High resolution multi-contrast cardiovascular magnetic resonance (CMR) is a noninvasive technique capable of providing detailed vessel wall information with high accuracy [12]. A number of studies have used multicontrast CMR as a reliable tool in the assessment of atherosclerotic lesions in various arterial beds, including the carotid, aorta and coronary [13-15]. One prior study demonstrated the reliability and reproducibility of measurement of atherosclerotic plaque volume in the superficial femoral artery [16]. However, to our knowledge, no prior CMR studies have reported on composition of atherosclerotic plaque in the lower extremity arteries.

In this study, we hypothesize that there is a correlation between plaque eccentricity and plaque morphology and composition. These plaque characteristics are measured using high-spatial-resolution multi-contrast CMR in the superficial femoral artery (SFA).

\section{Methods}

\section{Study population}

From February to July 2008, twenty-eight subjects were randomly selected from among participants in the Walking and Leg Circulation Study (WALCS) III cohort. WALCS III participants were identified from consecutive patients with PAD in the non-invasive vascular laboratories at Northwestern Memorial Hospital (Chicago, IL) or from consecutive patients with a diagnosis of PAD who were seen in the vascular surgery, general internal medicine, geriatric, cardiology, or endocrinology clinics at the Northwestern Medical Faculty Foundation (Chicago, IL).

The inclusion criterion was an ankle-brachial index $(\mathrm{ABI})<1.00$ at the baseline study visit. Exclusion criteria were contraindications to CMR, recent major surgery or cardiovascular event, inability to walk without a wheelchair, stopping during the six-minute walk test due to shortness of breath or arthritic symptoms, inability to speak English, nursing home residence, lower extremity limb amputation, dementia, life expectancy $<12$ months, and hepatorenal syndrome with associated renal insufficiency. Participant report was collected to acquire information on comorbidities, using established methods [17]. Comorbidities assessed were history of hypertension, diabetes, and coronary artery disease. Cigarette smoking history was measured by self-report. All participants gave informed written informed consent. The study was approved by the institutional review boards of participating sites.

\section{CMR protocol}

All MR scans were performed at a 1.5T (MAGNETOM Espree, Siemens) platform using four-element phasedarray surface coils. A standardized protocol (Table 1) was used to obtain 2D bright blood time-of-flight (TOF), black blood T1 weighted, T2 weighted and proton-density weighted (PD) images. Dual regional saturation bands (thickness: $50 \mathrm{~mm}$ ) $10 \mathrm{~mm}$ above and below the imaging volume were applied before data acquisition of each slice to suppress the luminal blood signal. Data for each slice were acquired in an interleaved fashion with each repetition time [18]. Fat suppression was applied in all black-blood sequences to improve identification of vessel outer wall boundaries as well as to avoid chemical shift artifacts. The longitudinal coverage of the superficial femoral artery (SFA) was about $40 \mathrm{~mm}$, beginning at the point where the common femoral artery (CFA) bifurcates into the proximal SFA. After acquisition, all raw data underwent zero-filled interpolation to a matrix size of $512 \times 512$ pixels.

\section{Image analysis}

The multi-contrast weighted cross-sectional images were matched using the bifurcation of CFA as a fiducial marker (Figure 1). Two trained reviewers (F. L. and X. Z.) with at least one year experience in CMR performed the image review with consensus opinion. Image quality (IQ) was assessed using a previously developed four-point scale (1 = poor quality, 4 = excellent) [19]. Images of occluded SFA or poor image quality were excluded due to unidentifiable lumen and vessel wall. For images with an image quality score of 2 or greater, the lumen area (LA) and total vessel area (TVA) were outlined using semi-automatic image analysis software (CASCADE) [20]. Wall area (WA = TVA-LA), mean $/$ maximum $/$ minimum wall thickness (MeanWT, MaxWT, MinWT) were derived from the LA and TVA outlines.

The eccentricity index for each location was calculated by the equation: (MaxWT - MinWT)/MaxWT (Figure 2). A lesion was defined as eccentric if the index was $\geq 0.5$ and as concentric if $<0.5$ [9].

Criteria for outlining intraplaque hemorrhage (IPH), lipid rich necrotic core (LRNC) and calcification (CA) were based on previously published carotid CMR criteria for imaging with multi-contrast techniques [12] (Figure 3 ). Relative content (\%) of components was calculated as (component area/wall area)*100.

For each location, lesion type was classified as type I through VIII according to modified American Heart Association (AHA) criteria [13]. Locations with a lesion 
Table 1: Basic scan parameters for the femoral artery imaging protocol at 1.5T

\begin{tabular}{|c|c|c|c|c|}
\hline \multirow[t]{3}{*}{ Parameters } & \multicolumn{4}{|c|}{ Scan type } \\
\hline & 1 & 2 & 3 & 4 \\
\hline & black-blood PDW & black-blood T1W & black-blood T2W & bright-blood TOF \\
\hline Sequence & TSE & TSE & TSE & GRE \\
\hline Image mode & $2 \mathrm{D}$ & $2 \mathrm{D}$ & $2 \mathrm{D}$ & $2 \mathrm{D}$ \\
\hline Scan plane & Axial & Axial & Axial & Axial \\
\hline TR, msec & 2160 & 620 & 2160 & 38 \\
\hline TE, msec & 5.7 & 5.7 & 51 & 8.67 \\
\hline FOV, $\mathrm{cm}$ & $12 \times 12$ & $12 \times 12$ & $12 \times 12$ & $12 \times 12$ \\
\hline Matrix size & $192 \times 192$ & $192 \times 192$ & $192 \times 192$ & $384 \times 384$ \\
\hline Resolution, $\mathrm{mm}^{2}$ & $0.63 \times 0.63$ & $0.63 \times 0.63$ & $0.63 \times 0.63$ & $0.31 \times 0.31$ \\
\hline Slice thickness, $\mathrm{mm}$ & 3 & 3 & 3 & 3 \\
\hline slices & 14 & 14 & 14 & 14 \\
\hline NEX & 10 & 12 & 10 & 2 \\
\hline Saturation band & on & on & on & off \\
\hline Fat suppression & Yes & Yes & Yes & No \\
\hline
\end{tabular}

TR: repetition time; TE: echo time; NEX: number of excitations

of type III and above were classified as diseased segments and were analyzed.

\section{Statistical analysis}

Statistical analysis was performed using SPSS for Windows (version 12.0, Chicago, IL) and $\mathrm{R}$ version 2.9.2. Quantitative data are presented as mean \pm standard deviation (SD). Qualitative data are presented as frequencies.

As the data involved multiple observations from each subject, Generalized Estimating Equation (GEE) models with robust standard error estimators were used to account for intra-subject correlations when performing hypothesis tests [21]. Differences in continuous variables between eccentric and concentric lesions were assessed using contrasts from normal linear regression GEE models. Differences in prevalence of plaque components between eccentric and concentric lesions were assessed using logistic regression GEE models. Except for regression coefficients, all summary statistics (means, standard deviations, frequencies, etc) are presented without any adjustment for intra-subject correlation.

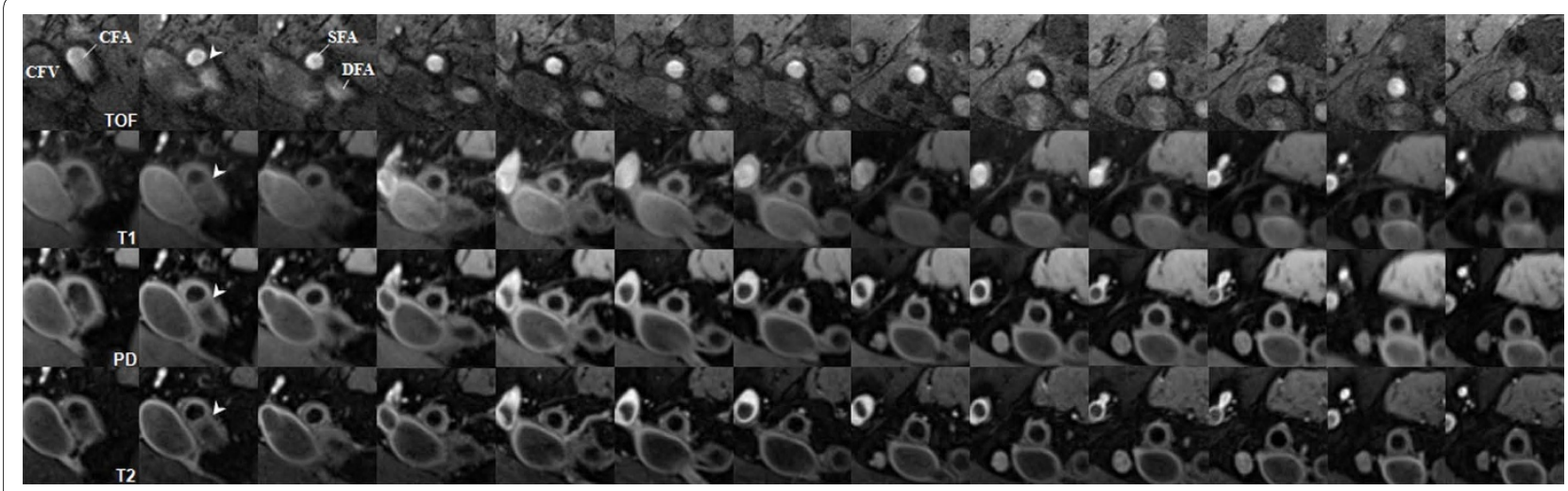

Figure 1 Example of cross-sectional CMR images with four contrast weightings. The multi-contrast weighted images were matched using bifurcation (arrowhead) of common femoral artery as a fiducial marker. (CFA: common femoral artery; CFV: common femoral vein; SFA: superficial femoral artery; DFA: deep femoral artery). 

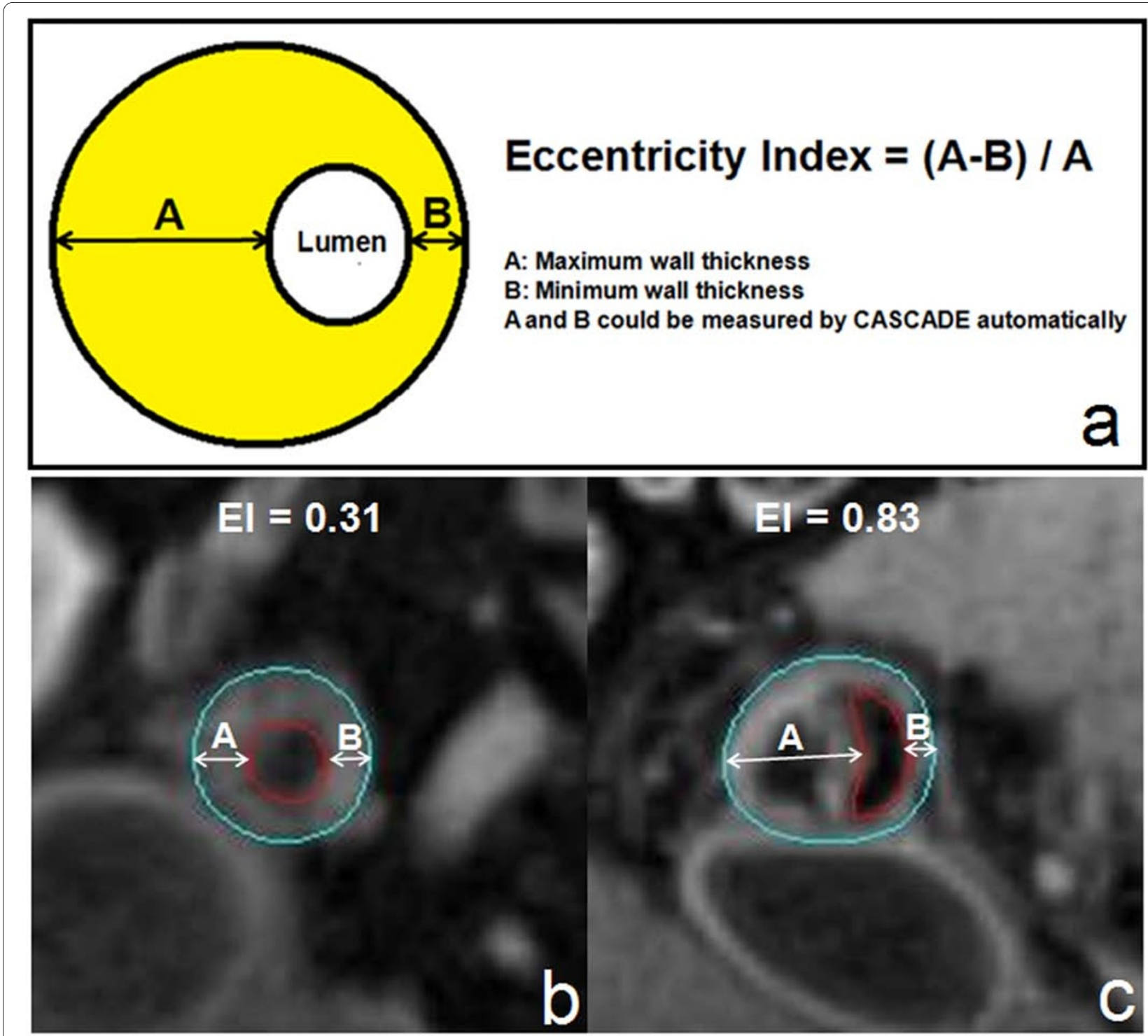

Figure 2 Delineation of eccentricity measurement. (a), Eccentricity Index = (A - B)/A. A (Maximum wall thickness) and B (Minimum wall thickness) can be measured by CASCADE automatically. The eccentric lesion is defined as El $\geq 0.5$, concentric lesion as El $<0.5$; (b) and (c), Examples of femoral arterial plaques with eccentricity index of 0.31 and 0.83 , respectively. A big calcification can be seen in the eccentric plaque (dark area). (blue: outer wall boundary; red: lumen).

Multivariate GEE logistic regression analysis was performed to correlate the prevalence of plaque components with lesion eccentricity after adjusting for atherosclerosis risk factors (age, gender, hypertension, diabetes, coronary artery disease, smoking and statin therapy) and wall area. All hypothesis tests were conducted within the context of a GEE model were two-sided, and considered significant when the P value was $<0.05$.

\section{Results}

After exclusion of two subjects with an occluded SFA and twenty-one locations with poor image quality (IQ < 2),
272 cross-sectional SFA images from 26 patients were analyzed. Demographic data for the study population are presented in Table 2.

Among the 272 locations, 180 locations had a lesion of type III or above, including 81 type III, 16 type IV-V and 83 type VII. According to the eccentricity index (EI), 115 locations were identified as eccentric lesions (eccentricity index $\geq 0.5$ ) and 65 were concentric lesions. In 18 subjects, eccentric and concentric lesions were coexistent in the same arteries. Two subjects showed only concentric and six showed only eccentric lesions. 


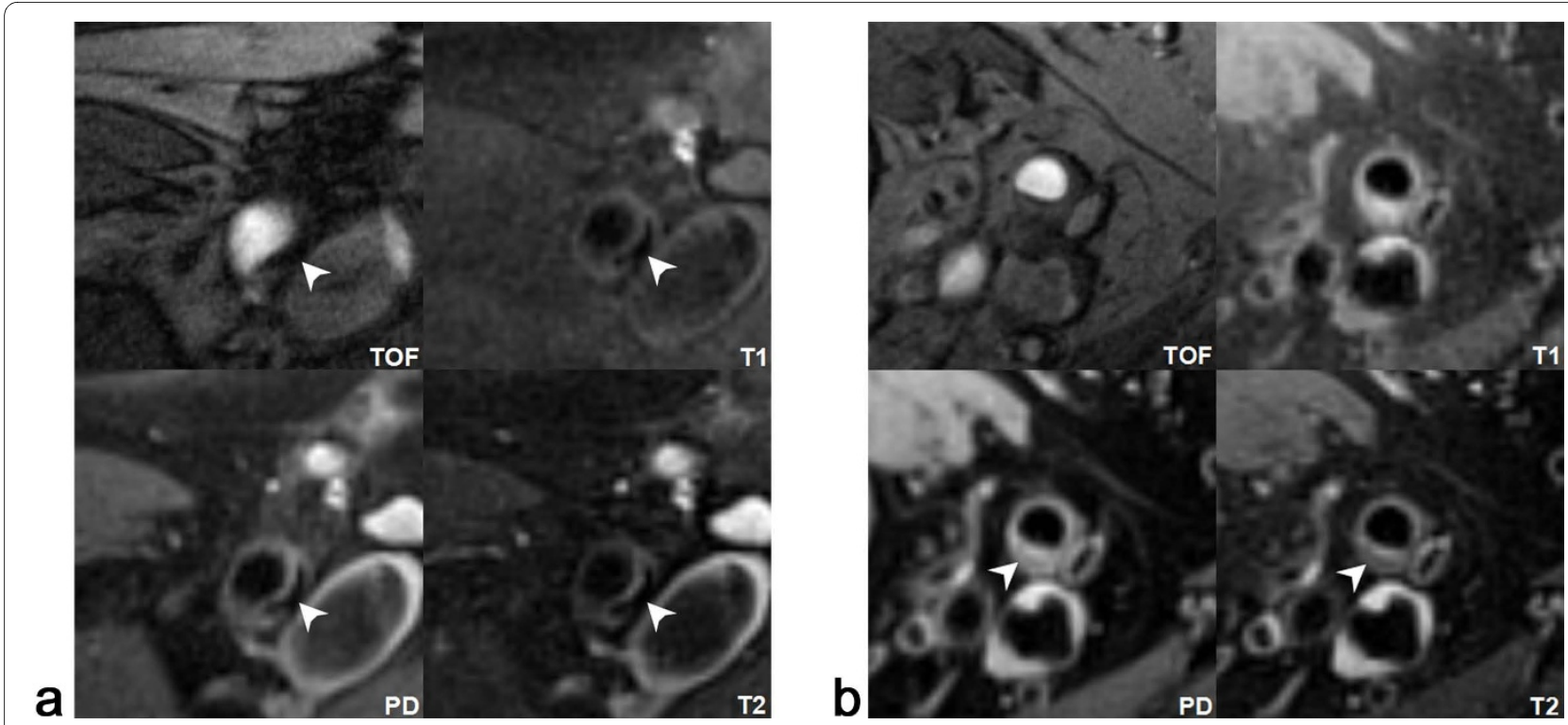

Figure 3 Example of signal pattern of calcification and lipid rich necrotic core (LRNC) in multi-contrast weighted CMR. (a) The calcification shows low intensity signal on all four contrast weightings (arrowhead) (b) The typical image pattern of LRNC is iso-intensity on TOF, iso/high-intensity on T1WI and low intensity on PD and T2WI. In the posterior vessel wall of superficial femoral artery, a crescent shaped LRNC can be identified (arrowhead).

The eccentric lesions exhibited significantly larger wall area, MeanWT, and MaxWT, while there were no significant differences in lumen area. Concentric lesions had larger MinWT (Table 3, Figure 4).

After adjusting for lumen area in a multivariate linear regression GEE model, eccentricity index was significantly associated with the wall area $(\mathrm{p}<0.001)$. On average, for a given lumen area, each 0.1 increase in eccentricity index was associated with a 9.3\% larger wall area.

No intraplaque hemorrhage was detected in these patients. The prevalence of LRNC in eccentric and con-

\section{Table 2: Demographic data in study population}

\begin{tabular}{ll}
\hline Parameter & Datum \\
\hline Age $(\mathbf{y})$ & \\
Height $(\mathbf{c m})$ & $73.62 \pm 8.22$ \\
Weight $(\mathbf{k g})$ & $165.73 \pm 8.67$ \\
Male & $77.53 \pm 16.86$ \\
History of hypertension & $54 \%(14 / 26)$ \\
History of diabetes & $38 \%(10 / 26)$ \\
History of coronary artery disease & $35 \%(9 / 26)$ \\
Active smoker & $46 \%(12 / 26)$ \\
Low density lipoprotein $(\mathbf{m g} / \mathbf{d l})$ & $12 \%(3 / 26)$ \\
High density lipoprotein $\mathbf{( m g / d l )}$ & $92.38 \pm 32.56$ \\
Current statin therapy & $51.46 \pm 18.70$ \\
Ankle-Brachial Index & $77 \%(20 / 26)$ \\
\hline
\end{tabular}

centric lesions was $13.0 \%(15 / 115)$ vs. $1.54 \%(1 / 65)(\mathrm{P}=$ 0.057). Differences in LRNC size were not considered because there was only a single concentric lesion with a LRNC. For calcification, the prevalence was 63.5\% (73/ $115)$ in eccentric cross-sections vs. $16.9 \%(11 / 65)$ in concentric cross-sections $(P<0.001)$. Considering only locations with calcification, eccentric lesions had more calcifications, both in terms of area and relative area $(\mathrm{p}=$ 0.002). Using multivariate logistic regression GEE models, the eccentricity (dichotomous as eccentric or concentric) was independently correlated to the presence of calcification (odds ratio 3.78, 95\% CI 1.47-9.70) after adjusting for atherosclerotic risk factors and wall area.

\section{Discussion}

The femoral artery hardly tapers and has no major side branches [22], and the atherosclerotic femoral artery could present very diffuse lesions along the whole artery with different eccentricity geometries, which make the femoral artery ideal for the investigation of lesion eccentricity by cross-sectional CMR.

To our knowledge, this is the first study to investigate the association between plaque eccentricity, morphology and components in femoral artery using high-spatial-resolution multi-contrast weighted CMR. Our results demonstrate that plaque composition and morphology are strongly associated with the magnitude of lesion eccentricity.

Pathophysiologic studies described eccentric geometry as a preservation mechanism to maintain the opening of 
Table 3: Comparison of plaque morphology and components between eccentric and concentric lesions

\begin{tabular}{|c|c|c|c|}
\hline \multicolumn{4}{|c|}{ Eccentricity } \\
\hline & $\begin{array}{c}\text { Eccentric } \\
\text { (mean } \pm \text { SD) }\end{array}$ & $\begin{array}{l}\text { Concentric } \\
\text { (mean } \pm \text { SD) }\end{array}$ & P Value \\
\hline Lumen area, $\mathbf{m m}^{2}$ & $15.3 \pm 8.35$ & $13.2 \pm 8.8$ & 0.078 \\
\hline Wall area, $\mathbf{m m}^{2}$ & $38.8 \pm 14.9$ & $26.4 \pm 10.5$ & 0.019 \\
\hline Mean wall thickness, $\mathrm{mm}$ & $1.98 \pm 0.61$ & $1.53 \pm 0.31$ & $<0.001$ \\
\hline Max wall thickness, $\mathrm{mm}$ & $3.39 \pm 1.45$ & $1.94 \pm 0.45$ & $<0.001$ \\
\hline Min wall thickness, $\mathrm{mm}$ & $1.02 \pm 0.19$ & $1.19 \pm 0.24$ & $<0.001$ \\
\hline Calcification, $\mathbf{m m}^{2 *}$ & $4.90 \pm 5.91$ & $1.76 \pm 0.55$ & 0.002 \\
\hline$\%$ Calcification* & $10.1 \pm 7.95$ & $5.40 \pm 1.34$ & 0.002 \\
\hline
\end{tabular}

* Only locations that exhibited calcification were included; $\mathrm{N}=73$ for eccentric, $\mathrm{N}=11$ for concentric.

$\%$ Calcification $=($ calcification area/Wall area $) \times 100$

lumen in the presence of atherosclerosis. Hemodynamic forces acting on the endothelium cells are important in the initiation of the atherosclerotic process and proatherogenic shear stress profiles produce different plaque

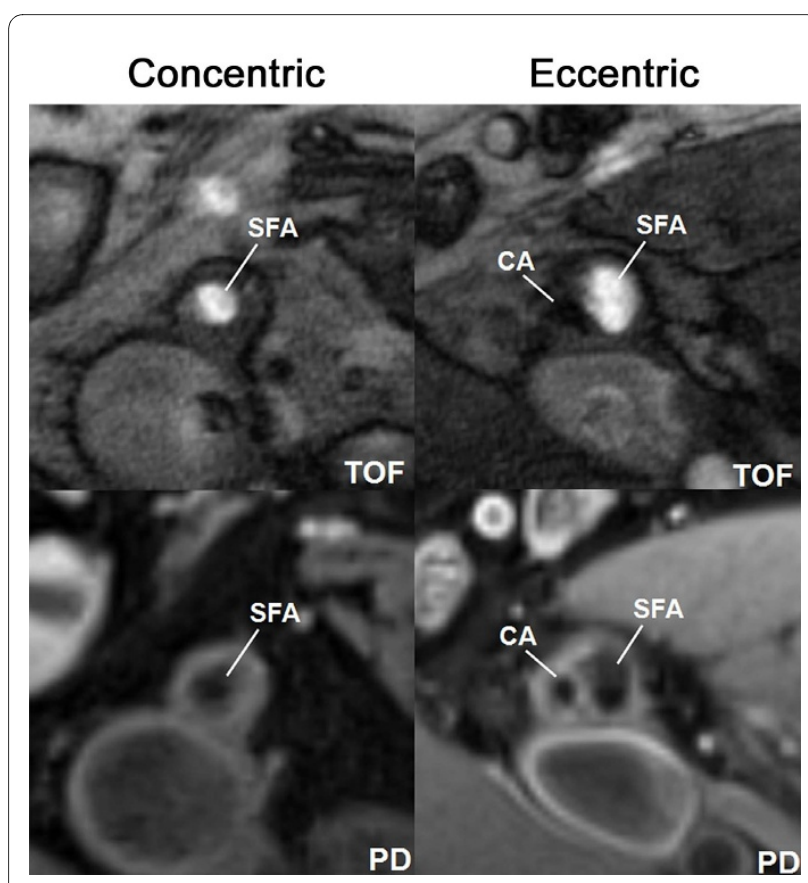

Figure 4 Example of concentric and eccentric lesions in multicontrast weighted CMR. Cross-sectional TOF and PD weighted images show examples of femoral arterial plaques with eccentricity index of 0.39 (concentric) and 0.78 (eccentric), respectively. The eccentric lesion has significant larger wall area $\left(27.50 \mathrm{~mm}^{2} \mathrm{vs} .15 .23 \mathrm{~mm}^{2}\right)$, total vessel area $\left(87.65 \mathrm{~mm}^{2}\right.$ vs. $\left.61.55 \mathrm{~mm}^{2}\right)$, maximum wall thickness $(5.09 \mathrm{~mm}$ vs. $2.27 \mathrm{~mm}$ ) and mean wall thickness ( $2.43 \mathrm{~mm}$ vs. $2.27 \mathrm{~mm}$ ), but still preserves larger lumen area $\left(27.50 \mathrm{~mm}^{2} \mathrm{vs} .15 .23 \mathrm{~mm}^{2}\right)$. A big piece of calcification (CA), which shows dark signal on all weightings, can be detected in eccentric plaque. phenotypes [23,24]. Low shear stress aggravates eccentric plaque build up, whereas oscillatory shear stress trends to induce small plaques with a concentric phenotype [24]. As a feedback-control loop, localized eccentric plaque distribution also contributes to normal lumen preservation [24], and, by consequence, a low shear stress distribution.

Lumen size measured by angiography has been applied as the gold standard for the evaluation of disease severity and pre-procedural risk assessment before interventions in coronary, carotid and femoral arterial diseases [25]. However, a number of necropsy studies have suggested that plaque size and vulnerability, other than stenosis, may also be important prognostic predictors for cardiovascular events $[26,27]$. In this study, the bigger plaques with unstable features, such as LRNC, exhibited lumen areas similar to the less advanced lesions due to the occurrence of eccentricity. Thus, a comprehensive assessment of lesion severity in atherosclerotic vessels may include not only the arterial stenosis but also the wall information.

When we controlled for lumen area, the eccentricity index was strongly correlated with wall area. For a fixed lumen area, a 0.1 increase in eccentricity index was associated with a $9.3 \%$ increase in wall area. Furthermore, the eccentric lesions trended to contain more LRNC (13.0\% vs. $1.54 \%$ in concentric), although this finding did not achieve statistical significance $(P=0.057)$. Our results demonstrate that the prevalence of LRNC is greater in eccentric lesions, compared to concentric lesions. This finding is in accordance with a previous clinical study regarding plaque eccentricity using intravascular ultrasound (IVUS) as image modality in coronary artery [9].

A number of histopathological studies have suggested that LRNC is a crucial determinant of the vulnerability of 
atherosclerotic lesions [28]. Accumulation of lipid content in eccentric lesion indicates this subgroup may be more likely to rupture and cause subsequent clinical events. T. Ohara, et al. [10] reported that, in patients with a carotid stenosis $>70 \%$, eccentric plaques were associated with a significantly increased incidence of ipsilateral stroke or transient ischemic attack compared to patients with concentric plaques, even though the degrees of arterial stenosis were identical. In a retrospective clinical study including 110 angina patients, Ambrose, et al. [29] found that eccentric lesions were more common in unstable angina than concentric lesions.

Although the clinical significances of calcification in coronary and carotid atherosclerosis are still controversial [30], the calcification in lower extremity arteries has been demonstrated to be strongly associated with more advanced PAD [31]. Guzman et al. [7] reported that calcification content in the tibial artery had the potency to be a better predictor of near-term risk of amputation than a low ABI in patients with PAD. In this study, after adjustment for the atherosclerotic risk factors and wall area in the multivariate logistic regression analysis, eccentricity was independently correlated to prevalence of calcification (odds ratio 3.78). The greater degree of calcification in eccentric lesion implies that the atherosclerotic femoral arteries involved with more eccentric lesions may be related to a worse prognosis than the arteries dominated by concentric lesions despite similar stenosis.

\section{Limitations}

Due to the insufficiency of surface coil coverage, only the cross-sectional MR images in proximal segments (about $40 \mathrm{~mm}$ ) of SFA were acquired and analyzed in this study. The lesions localized in this segment may not represent the overall plaque characteristics of the entire SFA. A 3D CMR sequence with larger coverage and suitable surface coil may provide more comprehensive imaging than the methods used in the current study. However, the methods reported here are state-of-the-art methods for measuring atherosclerotic plaque in the femoral arteries, and further work is necessary before 3D imaging can be used successfully to image atherosclerotic plaques in the superficial femoral artery in PAD patients. In addition, the sample size was small in this study. Further studies in a larger population are needed before these results can be generalized to the population at large.

\section{Conclusions}

The eccentricity of atherosclerotic plaque in the superficial femoral artery preserves lumen size and is associated with advanced plaque features in vessel wall, such as larger plaque burden, more lipid content and increased calcification. Further longitudinal study is needed to identify the prognostic significance of plaque eccentricity in the superficial femoral artery.

\section{Competing interests}

The authors declare that they have no competing interests.

\section{Authors' contributions}

FL conceived and designed the study, participated the data analysis and draft of the manuscript. MMM obtained funding, was involved in the study design of non-CMR data collection methods, provided oversight to all aspects of nonCMR data collection, and oversaw subject recruitment. DH gave a major contribution to the data. DL and TJC were responsible for obtaining funding, the CMR protocol and image acquisition. $\mathrm{XH}$ was involved in the image analysis. CMK, TSH and JW helped in coordinating the study and contributed to the manuscript draft. YC participated in the study design, assisted with obtaining funding, data analysis and the revision of the manuscript. All authors read and approved the final manuscript.

\section{Acknowledgements}

This study is supported by the National Heart Lung and Blood Institute (grants R01-HL083064).

\section{Author Details}

1Department of Radiology, Peking University First Hospital, Beijing, China, 2Department of Radiology, University of Washington, Seattle, WA, USA, ${ }^{3}$ Department of Medicine, Northwestern University Feinberg School of Medicine, Chicago, II, USA, ${ }^{4}$ Department of Radiology, Northwestern University Feinberg School of Medicine, Chicago, II, USA, ${ }^{5}$ Departments of Radiology and Medicine, University of Virginia, Charlottesville, VA, USA, 6 Department of Surgery, University of Washington, Seattle, WA, USA and ${ }^{7}$ Clinical Sites Research Program, Philips Research North America, Briarcliff Manor, NY, USA

Received: 21 January 2010 Accepted: 1 July 2010

Published: 1 July 2010

\section{References}

1. Hirsch AT, Criqui MH, Treat-Jacobson D, Regensteiner JG, Creager MA, Olin JW, Krook SH, Hunninghake DB, Comerota AJ, Walsh ME, McDermott MM, Hiatt WR: Peripheral arterial disease detection, awareness, and treatment in primary care. JAMA 2001, 286:1317-1324.

2. Criqui MH, Langer RD, Fronek A, Feigelson HS, Klauber MR, MCCann TJ, Browner D: Mortality over a period of 10 years in patients with peripheral arterial disease. N Engl J Med 1992, 326:381-386.

3. Cimminiello C: PAD. Epidemiology and pathophysiology. Thromb Res 2002, 106:V295-301.

4. Lumsden AB, Rice TW, Chen C, Zhou W, Lin PH, Bray P, Morrisett J, Nambi $\checkmark$, Ballantyne C: Peripheral arterial occlusive disease: magnetic resonance imaging and the role of aggressive medical management. World J Surg 2007, 31:695-704.

5. Takaya N, Yuan C, Chu B, Saam T, Underhill H, Cai J, Tran N, Polissar NL, Isaac C, Ferguson MS, Garden GA, Cramer SC, Maravilla KR, Hashimoto B, Hatsukami TS: Association between carotid plaque characteristics and subsequent ischemic cerebrovascular events: a prospective assessment with MRI--initial results. Stroke 2006, 37:818-823.

6. Davies MJ, Thomas AC: Plaque fissuring--the cause of acute myocardial infarction, sudden ischaemic death, and crescendo angina. Br Heart $J$ 1985, 53:363-373

7. Guzman RJ, Brinkley DM, Schumacher PM, Donahue RM, Beavers H, Qin X: Tibial artery calcification as a marker of amputation risk in patients with peripheral arterial disease. J Am Coll Cardio/ 2008, 51:1967-1974.

8. Mintz GS, Popma JJ, Pichard AD, Kent KM, Satler LF, Chuang YC, DeFalco RA, Leon MB: Limitations of angiography in the assessment of plaque distribution in coronary artery disease: a systematic study of target lesion eccentricity in 1446 lesions. Circulation 1996, 93:924-931.

9. Yamagishi M, Terashima M, Awano K, Kijima M, Nakatani S, Daikoku S, Ito K, Yasumura Y, Miyatake K: Morphology of vulnerable coronary plaque: insights from follow-up of patients examined by intravascular ultrasound before an acute coronary syndrome. J Am Coll Cardiol 2000, 35:106-111. 
10. Ohara T, Toyoda K, Otsubo R, Nagatsuka K, Kubota Y, Yasaka M, Naritomi H, Minematsu K: Eccentric stenosis of the carotid artery associated with ipsilateral cerebrovascular events. AJNR Am J Neuroradio/ 2008, 29:1200-1203

11. Nair P, Gruberg L, Beyar R: The eccentric lumenology. Acute Card Care 2006, 8:87-94.

12. Saam T, Ferguson MS, Yarnykh VL, Takaya N, Xu D, Polissar NL, Hatsukami TS, Yuan C: Quantitative evaluation of carotid plaque composition by in vivo MRI. Arterioscler Thromb Vasc Biol 2005, 25:234-239.

13. Cai JM, Hatsukami TS, Ferguson MS, Small R, Polissar NL, Yuan C: Classification of human carotid atherosclerotic lesions with in vivo multicontrast magnetic resonance imaging. Circulation 2002, 106:1368-1373

14. Kramer CM, Cerilli LA, Hagspiel K, DiMaria JM, Epstein FH, Kern JA: Magnetic resonance imaging identifies the fibrous cap in atherosclerotic abdominal aortic aneurysm. Circulation 2004, 109:1016-1021.

15. Sun B, Giddens DP, Long R Jr, Taylor WR, Weiss D, Joseph G, Vega D, Oshinski JN: Characterization of coronary atherosclerotic plaque using multicontrast MRI acquired under simulated in vivo conditions. J Magn Reson Imaging 2006, 24:833-841.

16. Isbell DC, Meyer CH, Rogers WJ, Epstein FH, DiMaria JM, Harthun NL, Wang $\mathrm{H}$, Kramer CM: Reproducibility and reliability of atherosclerotic plaque volume measurements in peripheral arterial disease with cardiovascular magnetic resonance. J Cardiovasc Magn Reson 2007, 9:71-76.

17. McDermott MM, Ades P, Guralnik JM, Dyer A, Ferrucci L, Liu K, Nelson M, Lloyd-Jones D, Van Horn L, Garside D, Kibbe M, Domanchuk K, Stein JH, Liao Y, Tao H, Green D, Pearce WH, Schneider JR, McPherson D, Laing ST, McCarthy WJ, Shroff A, Criqui MH: Treadmill exercise and resistance training in patients with peripheral arterial disease with and without intermittent claudication: a randomized controlled trial. JAMA 2009, 301:165-174

18. Koktzoglou I, Chung YC, Mani V, Carroll TJ, Morasch MD, Mizsei G, Simonetti OP, Fayad ZA, Li D: Multislice dark-blood carotid artery wall imaging: a 1.5 T and 3.0 T comparison. J Magn Reson Imaging 2006, 23:699-705.

19. Underhill HR, Yarnykh VL, Hatsukami TS, Wang J, Balu N, Hayes CE, Oikawa $\mathrm{M}, \mathrm{Yu}$ W, Xu D, Chu B, et al:: Carotid plaque morphology and composition: initial comparison between 1.5- and 3.0-T magnetic field strengths. Radiology 2008, 248:550-560

20. Kerwin W, Xu D, Liu F, Saam T, Underhill H, Takaya N, Chu B, Hatsukami T, Yuan C: Magnetic resonance imaging of carotid atherosclerosis: plaque analysis. Top Magn Reson Imaging 2007, 18:371-378.

21. Zeger SL, Liang KY: Longitudinal data analysis for discrete and continuous outcomes. Biometrics 1986, 42:121-130.

22. Vink A, Schoneveld AH, Richard W, de Kleijn DP, Falk E, Borst C, Pasterkamp G: Plaque burden, arterial remodeling and plaque vulnerability: determined by systemic factors? J Am Coll Cardiol 2001, 38:718-723.

23. Davies PF: Mechanisms involved in endothelial responses to hemodynamic forces. Atherosclerosis 1997, 131(Suppl):S15-17.

24. Helderman F, Segers D, de Crom R, Hierck BP, Poelmann RE, Evans PC, Krams R: Effect of shear stress on vascular inflammation and plaque development. Curr Opin Lipido/ 2007, 18:527-533.

25. Just H: Coronary arteriography: current technique and standards of equipment. Med Prog Technol 1977, 5:119-125.

26. Kullo IJ, Edwards WD, Schwartz RS: Vulnerable plaque: pathobiology and clinical implications. Ann Intern Med 1998, 129:1050-1060.

27. Shin J, Edelberg JE, Hong MK: Vulnerable atherosclerotic plaque: clinical implications. Curr Vasc Pharmacol 2003, 1:183-204.

28. Virmani R, Burke AP, Farb A, Kolodgie FD: Pathology of the vulnerable plaque. J Am Coll Cardio/ 2006, 47:C13-18.

29. Ambrose JA, Winters SL, Stern A, Eng A, Teichholz LE, Gorlin R, Fuster V: Angiographic morphology and the pathogenesis of unstable angina pectoris. J Am Coll Cardiol 1985, 5:609-616

30. Abedin M, Tintut Y, Demer LL: Vascular calcification: mechanisms and clinical ramifications. Arterioscler Thromb Vasc Biol 2004, 24:1161-1170.

31. Niskanen L, Siitonen O, Suhonen M, Uusitupa MI: Medial artery calcification predicts cardiovascular mortality in patients with NIDDM. Diabetes Care 1994, 17:1252-1256. doi: $10.1186 / 1532-429 X-12-37$

Cite this article as: Li et al., The association of lesion eccentricity with plaque morphology and components in the superficial femoral artery: a high-spatial-resolution, multi-contrast weighted CMR study Journal of Cardiovascular Magnetic Resonance 2010, 12:37

\section{Submit your next manuscript to BioMed Central and take full advantage of:}

- Convenient online submission

- Thorough peer review

- No space constraints or color figure charges

- Immediate publication on acceptance

- Inclusion in PubMed, CAS, Scopus and Google Scholar

- Research which is freely available for redistribution
C Biomed Central 